
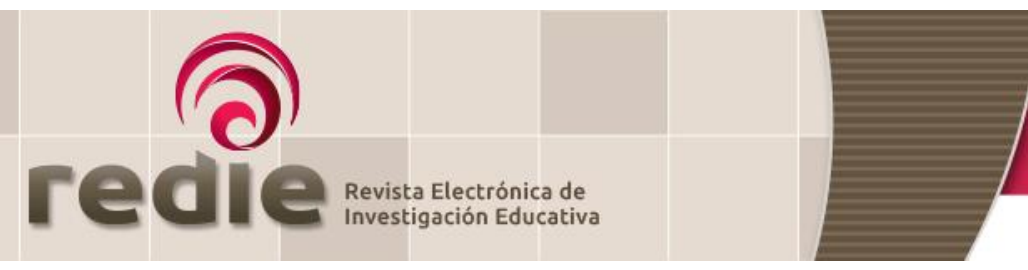

\title{
La construcción de conocimiento pedagógico de los profesores en formación durante el prácticum
}

\section{Pre-Service Teachers' Practical Knowledge: Learning to Teach during the Practicum Experience}

Juanjo Mena Marcos (*) juanjo mena@usal.es

Raquel Gómez Sánchez (*) raquelgs@usal.es

María Luisa García Rodríguez (*) malugaro@usal.es

(*) Universidad de Salamanca

(Recibido: 21 de febrero de 2017; Aceptado para su publicación: 29 de septiembre de 2017)

Cómo citar: Mena, J., Gómez, R. y García, M. L. (2019). La construcción de conocimiento pedagógico de los profesores en formación durante el prácticum. Revista Electrónica de Investigación Educativa, 21, e27, 1-13. doi:10.24320/redie.2019.21.e27.1831

\section{Resumen}

En este trabajo se presenta parte de los resultados del Proyecto Europeo K1 ACTTEA (2012-2015) en el cual participaron España, Estonia, Finlandia y Holanda. El objetivo fue valorar qué tipos de conocimiento pedagógico asimilan los profesores durante el prácticum. En los datos recogidos en España se llevó a cabo un estudio empírico siguiendo tres fases: grabación de sesiones de clase, análisis de incidentes críticos y elaboración de informes individuales. Se emplearon tres condiciones en el diseño de investigación: reflexión individual, diálogo entre compañeros y diálogo con profesor-tutor. Se siguió un procedimiento inductivo basado en el análisis temático y proposicional y se utilizó la prueba Chi cuadrado y V de Cramer. Los resultados evidencian que los profesores en formación bajo la condición C son más capaces de aprender un conocimiento pedagógico sofisticado y generalizable, mientras que las condiciones $\mathrm{A}$ y $\mathrm{B}$ permiten entender mejor lo que hacen en la práctica.

Palabras clave: Prácticum, formación del profesorado, tutoría, reflexión docente, conocimiento práctico.

\section{Abstract}

In this paper we present some results of the European Project Erasmus K1 ACTTEA (2012-2015) in which four countries participated (Estonia, Finland, The Netherlands and Spain). The objective of the project was to assess what kind of knowledge pre-service teachers learn when teaChing in the Practicum setting. An empirical study was conducted in each country following three phases. video recordings were made in sessions; analysis of critical incidents; and an individual report of learning was performed. Three conditions were established: Individual reflection; Dialogue among peers; and Dialogue with a tutor. An inductive procedure based on propositional and thematic analysis was followed, and the Chi Square Test and V Cramer were used. Results from the Spanish data show that student teachers under the condition $\mathrm{C}$ are better able to learn a kind of sophisticated and generalizable knowledge, while conditions $\mathrm{A}$ and $\mathrm{B}$ allow students to better understand what they had done. 


\section{Introducción}

El profesorado es un elemento determinante para una buena calidad educativa, por ello el sistema educativo debe atender a la formación de los docentes (Cerezo, Ortega y Molina, 2016). La preocupación por la formación del profesorado ha ido en aumento en los últimos años, lo que ha provocado que cada vez más los programas de formación incluyan resultados de investigación, contenidos prácticos y estrategias docentes dentro de sus planes de estudio (Grau, Gómez y Perandones, 2009). Como parte de esa formación se incluye el prácticum en la formación inicial del profesorado, donde se ofrece la oportunidad de aplicar en las escuelas los conocimientos y competencias adquiridos en el grado universitario. A través del prácticum se pretende estudiar el proceso de aprendizaje que desarrolla el profesorado en formación durante la fase de inmersión en los contextos escolares. Los profesores deben ser conscientes de las responsabilidades que tienen y adquirir las competencias necesarias para intervenir en contextos educativos (Fernández, Barrio, De Cisneros, Rodríguez y Rivas, 2015), por ello existe un interés creciente por identificar las estrategias más eficaces para avanzar hacia una mayor calidad y eficiencia en la preparación del personal docente (Mena, Hennissen y Loughran, 2017).

La formación práctica de un profesor en formación es de relevancia tanto para el desempeño de su profesión como para su desarrollo personal y académico (Raposo y Zabalza, 2011), pero el hecho de realizar unas prácticas sin análisis ni reflexión no implica una mejora automática del conocimiento profesional (Zeichner, 2007).

Durante el prácticum los alumnos deben adquirir las competencias para poder desarrollar las actividades de carácter profesional, participando en todo el proceso docente y colaborando con la comunidad educativa (Fernández, Gértrudix, De Cisneros, Rodríguez y Rivas, 2015). Por ello es importante llevar a cabo una reflexión sobre la práctica docente, ya que es un medio para estimular el aprendizaje y adquirir las distintas competencias.

Рara reforzar los procesos de aprendizaje del profesorado los programas de formación recurren a la reflexión como método de análisis, a través de ella los maestros observan y consideran sobre su práctica para generar conocimiento sobre la realidad educativa (Beltrán, 2003). La reflexión refuerza los procesos profesionales de aprendizaje (Dewey, 1933; Hatton y Smith, 1995; Schön, 1983) de tal manera que permite a los profesores pasar de una experiencia a otra adquiriendo mayor comprensión.

Según Elliott (1993), la investigación-acción "es un estudio de una situación social con el fin de mejorar la calidad de la acción dentro de la misma" (p. 88). Por lo tanto, como destacan Kemmis y McTaggart (1988), la investigación-acción permite mejorar la educación mediante la investigación de los procesos del aula, lo que genera un conocimiento profesional pedagógico en y para la práctica (Clandinin, 1985; Elbaz, 1981).

Husu, Toom y Patrikainen (2008) han diseñado el procedimiento de reflexión guiada como un modelo que ayude a los profesores en formación a identificar los incidentes críticos que se suceden en la práctica profesional.

Los incidentes críticos se entienden como eventos importantes que se producen en una situación dada. Sólo se someten a análisis cuando hacen referencia a los acontecimientos particulares que son importantes para el estudiante y el tutor (Orland-Barack, 2005). Los eventos críticos pueden sustentar las experiencias positivas o negativas de enseñanza (Husu et al., 2008) y desencadenar conversaciones significativas de tutoría en las que se hacen preguntas acerca de cuándo, qué y por qué sucedió (Carnot y Stewart, 2006).

Los incidentes críticos que se pueden identificar tienen relación con: el profesor, el estudiante y el contenido (Patrikainen, 2008; Toom, 2006). De esta manera se pueden establecer cuatro tipos de incidentes críticos: RP = relación pedagógica (entre profesor y alumno); RC = relación de contenido (entre el profesor y el contenido); RA = relación de aprendizaje (entre el estudiante y el contenido); RD = relación didáctica (entre el profesor y el aprendizaje del estudiante). 
Otro procedimiento conocido para reflexionar sobre la práctica sería el modelo ALACT $^{1}$ de Korthagen (2001), que consta de 5 pasos: 1. Acción, 2. Revisar la acción, 3. Tomar conciencia sobre aspectos esenciales, 4. Crear métodos de acción alternativo y 5. Ensayo. Ambos pueden ejecutarse de dos formar distintas: individual o dialógico (mediante compañero o tutor). Esta reflexión sobre la práctica será beneficiosa si se incita a los futuros profesores a reflexionar sobre su propia práctica y si se estimula el aprendizaje reflexivo entre pares (Korthagen y Lagerwerf, 1999).

Además, los comentarios o feedback que proporciona el tutor son elementos claves en el proceso de la reflexión de los futuros docentes (Serrat y Badia, 2015) ya que proporciona apoyo, estímulo y asesoramiento a los profesores en formación, lo que hace que se sientan valorados y cómodos con ellos mismos como maestros (Koballa y Glynn, 2007). Por lo tanto, la reflexión con el tutor debería ser una oportunidad para contrastar orientaciones hacia el aprendizaje y considerar todos los ámbitos de su aprendizaje (Korthagen, 1999).

\subsection{El conocimiento pedagógico como elemento del conocimiento práctico}

El conocimiento práctico puede definirse como el conjunto de saberes profesionales que el profesorado desarrolla a partir de su experiencia práctica y que son usados de manera recurrente sobre dicha práctica (Cuevas, 2013).

Según Mishra y Koehler (2006), el conocimiento práctico se divide en: conocimiento de contenido, conocimiento pedagógico y conocimiento tecnológico. En este artículo nos centramos en estudiar el conocimiento pedagógico, es decir, el que se refiere al conjunto de estrategias y principios didácticos que ayudan a los profesores en formación a entender mejor su práctica como docentes.

Siguiendo a Mena, García y Tillema (2008) y Mena, García, Clarke y Barkatsas (2016), son evidentes cuatro tipos de conocimientos profesionales en las conversaciones con los tutores: 1) Los recuerdos son reproducciones directas de la práctica en forma de eventos o incidentes; por ejemplo: "Los niños fueron capaces de hacer todos los experimentos con los padres"; 2) Valoraciones: constituyen juicios de valor sobre la acción que está siendo recordada. Se pueden dividir en: valoraciones positivas (por ejemplo: "Los padres estaban entusiasmados") y evaluaciones negativas (por ejemplo, "La actividad llevó demasiado tiempo"); 3) Reglas: principios prácticos o estrategias metodológicas que los maestros extraen de sus experiencias (Elbaz, 1981), por ejemplo, "Hay que adaptar el uso del lenguaje para la edad de los alumnos"; 4) Los artefactos: son instrumentos conceptuales, soportes físicos y herramientas que utilizan los maestros en acción (Shulman, 2002) sobre la base de sus generalizaciones de la experiencia y que pueden ayudar a la práctica futura, por ejemplo, "La comprensión del tema de historia de hoy mejora con una breve dramatización".

Los cuatro tipos de conocimiento se pueden agrupar en dos niveles de complejidad: recuerdos y valoraciones se caracterizan como un primer nivel de conocimiento narrativo (conocimiento específico situación que consiste en la estimulación de los acontecimientos y episodios de la práctica), mientras que las reglas y artefactos constituyen un segundo nivel de conocimiento inferencial (Bruner 1991), un conocimiento más sofisticado a través del cual se da mucha información abstracta (es decir, los principios de la práctica) que se generaliza a partir del contexto en cuestión.

Además, encontramos justificaciones prácticas que se refieren a los argumentos prácticos que dan los profesores para entender sus experiencias, y justificaciones teóricas que son argumentos teóricos que los profesores utilizan para apoyar lo que hacen en la práctica (Toom, 2012).

El objetivo principal de esta investigación es describir cómo los profesores en formación aprenden de su propia práctica mediante la grabación de sus acciones en video, el visionado crítico con pares y el comentario de las acciones realizadas con los mentores (profesores tutores de la escuela). De modo más

\footnotetext{
${ }^{1}$ Por sus siglas en inglés: Action, Looking back on action, Awareness of Essentials aspects, Creating alternative methods of actions and Trial.
} 
concreto, los objetivos que se plantean en esta investigación son:

1) Describir qué tipos de conocimientos pedagógicos surgen en la práctica docente de los profesores en formación a partir de la reflexión sobre su práctica.

2) Comparar qué condición de reflexión: individual, reflexión con compañeros y con el profesortutor favorece la elicitación y asimilación de conocimientos más sofisticados.

\section{Método}

La investigación parte de un paradigma interpretativo, ya que el propósito es entender las experiencias e interacciones entre los profesores en formación y tutores de prácticas (Cohen y Manion, 1994). Se utilizó un análisis de contenido para comparar las tres condiciones de reflexión.

El estudio se llevó a cabo en el marco del Proyecto Europeo ACTTEA, financiado por la Unión Europea (2012-2015), en el cual participaron España, Estonia, Finlandia y Holanda. Durante el año 2014 se seleccionaron en España 88 estudiantes del grado de Educación Primaria de la Facultad de Educación en la Universidad de Salamanca. Para revelar el tipo de conocimiento que surge en la práctica educativa como docente se llevaron a cabo grabaciones ${ }^{2}$ de una sesión impartida por el alumno y posteriormente se reflexionó individualmente, con un compañero y con el tutor de prácticas (mentor).

Entre los participantes ( $n=88$ ) se incluyen los alumnos del grado de Primaria de los años académicos 2012/2013 y 2013/2014, así como 33 tutores de los colegios que tutorizaron a los alumnos en prácticas.

La recogida de datos se realizó en dos fases: en una primera se seleccionaron 20 alumnos matriculados en 2013 y en la segunda fase participaron 68 alumnos del 2014, todos recibieron un curso de formación de 18 horas. En una segunda fase se invitó a los participantes a seguir el procedimiento de reflexión guiada (Husu et al., 2008). Según este procedimiento, en un primer momento se visiona la sesión del profesor en formación; después de dos días los profesores en formación seleccionan dos incidentes críticos (uno positivo y otro negativo), en ese momento los alumnos reflexionan sobre lo sucedido en la clase respondiendo a las preguntas: “Qué está pasando durante la sesión?, ¿cuáles son los eventos más significativos y por qué?" Una semana después se realiza una reflexión oral, es decir, un diálogo entre compañeros (dos profesores en formación) o un diálogo entre el profesor en formación y el profesor tutor (supervisor de prácticas). En estas conversaciones se hacen preguntas como: "¿Qué sucedió en este incidente?, ¿por qué el incidente es importante para ti?, ¿cuál es el significado general de este incidente?" Por último, entre una o dos semanas después profesores en formación realizan una reflexión escrita dando respuestas a preguntas del tipo: "¿Qué has aprendido de todo esto?, ¿cuál es la relación del incidente crítico con la teoría?, ¿cómo actuarías en una situación similar en el futuro?".

\subsection{Análisis de los datos}

En la investigación se utilizó una metodología mixta partiendo de un análisis cualitativo inicial que permitió obtener información con un mayor grado de detalle (Binda y Balbastre-Benavent, 2013). Se hizo una codificación temática para las transcripciones, se clasificaron los temas encontrados y se compararon los tres tipos de reflexiones encontrando algunas temáticas similares y otras diferentes. Luego se cuantificaron las unidades lingüísticas de las conversaciones y reflexiones docentes. Se procedió a un triple análisis de menor a mayor grado de escrutinio en función de la amplitud de la unidad de análisis: incidentes críticos (más amplios), temas, proposiciones (más analíticos) (Mena, García, Gómez y Ramírez, 2016). (ver Anexo).

Análisis de incidentes críticos. Los profesores en formación eligieron el evento positivo y negativo más significativo de su sesión práctica para posteriormente reflexionar sobre él. Una vez elegidos los dos

\footnotetext{
${ }^{2}$ Todas las grabaciones se llevaron a cabo respetando las normas éticas.
} 
incidentes dos analistas procedieron a dividir el diálogo en unidades de pensamiento, esto es, oraciones temáticas que resumen el contenido del incidente positivo o negativo y que se clasifican en torno a cuatro categorías: (Johnson, 1995; Patrikainen, 2008; Toom, 2006): Relación Pedagógica (RP): el incidente refleja la relación entre el profesor y el estudiante; Relación de Contenido (RC): relación entre el profesor y el contenido; Relación de Aprendizaje (RA): relación entre el estudiante y el contenido; Relación Didáctica (RD): relación entre el profesor y el aprendizaje del estudiante.

Se llevó a cabo un análisis de fiabilidad en el que se seleccionaron 15 unidades al azar y se categorizaron por dos jueces distintos. El coeficiente Kappa de Cohen fue de $k=0.73$

Análisis temático. El análisis temático es una técnica que permite reducir información primaria en diferentes niveles de categorías siguiendo un procedimiento de análisis fundamentado (Strauss y Corbin, 1994). Inicialmente partimos de tres macrocategorías identificadas en los incidentes críticos: profesor en formación, alumnos del aula y contenido. De acuerdo a esta clasificación se van incluyendo los subtemas que se encuentran dentro de las sesiones grabadas. Una vez obtenido el sistema de categorías y refinado por sucesivas aproximaciones se procedió a establecer el análisis de fiabilidad con otras 15 unidades. El coeficiente Kappa fue de $\mathrm{k}=0.85$

Análisis proposicional. El análisis proposicional es una técnica que permite dividir el discurso en las ideas básicas que se comparten en las interacciones (Tierney y Mosenthal, 1983). Una proposición es, en esencia, una idea del texto que contiene un único predicado (Kieras y Bovair, 1984). El texto resultante de las reflexiones se divide en proposiciones, una vez dividido se clasifican en cuatro tipos de conocimiento profesional: recuerdos, valoraciones, reglas y artefactos. Los dos primeros son considerados conocimiento narrativo, y los dos últimos como conocimiento inferencial. Además, clasificamos las proposiciones según los códigos SA (cuando habla el tutor) y ST (cuando habla el alumno en prácticas), y así comprobar por quién es inducido el conocimiento profesional.

En este apartado no se llevó a cabo un análisis de fiabilidad, ya que el análisis proposicional ha sido probado en estudios anteriores (Mena, Sánchez y Tillema, 2008; Mena, García, Clarke y Barkatsas, 2016) con índices superiores a $k=0.80$

Por último, para comparar las tres condiciones se realizó un análisis cuantitativo, el cual se llevó a cabo sobre la base de los conteos de frecuencias junto con la prueba no paramétrica (Chi cuadrado) utilizando la V de Cramer (Cohen, 1998) con el fin de evaluar la existencia y magnitud de una posible asociación significativa entre: a) la participación de la tutoría y la obtención de conocimientos prácticos; b) la directividad y los conocimientos profesionales; c) el papel del tutor de prácticas y el conocimiento profesional. La $\vee$ de Cramer es una corrección que se aplica al coeficiente Chi cuadrado que oscila entre $0=n o$ asociación y 1=fuerte asociación. (Rodríguez, 2001).

\section{Resultados}

En este apartado se muestran los resultados que dan respuesta al tipo de conocimiento pedagógico que surge en la práctica docente de los profesores en formación a partir de la reflexión sobre su práctica; y nos ayudarán a comparar qué condición de reflexión favorece la elicitación y asimilación de conocimientos más sofisticados y más útiles para la profesión.

\subsection{Tipos de conocimientos pedagógicos que emanan de la práctica de los profesores en formación}

En primer lugar, en el análisis de incidentes críticos fueron analizadas 1,197 unidades de pensamiento, en las cuales se seleccionaron los incidentes positivos y negativos de la sesión. Un $60.56 \%$ de los incidentes eran positivos y un $39.43 \%$ fueron negativos, esto se debe a que los alumnos consideraban más fácil mencionar los aspectos positivos que los negativos. 
El conocimiento práctico que extraen los alumnos consiste en la interacción de tres elementos importantes: maestro, alumnos y contenido. En total se extraen 1,514 oraciones temáticas divididas en las tres reflexiones (individual, con compañero y con tutor). De ellas, $4.62 \%$ están relacionadas con el profesor y el alumno (RP), 5.95\% están relacionas con el profesor y el contenido (RC), $0.53 \%$ trata sobre el alumno y el contenido (RA), y 88.90\% se obtiene en la relación didáctica.

En el análisis temático se obtuvieron tres macrotemas: docencia, aprendizaje de los alumnos y contenidos académicos. Cada macrotema dio lugar a otros temas situados en tres niveles de jerarquía. Los profesores en formación aludieron más al primero de ellos: la docencia, representando un 64.6\% del total. Dentro de este macrotema se reflejaron otros temas referidos mayoritariamente a la instrucción (62.3\%) y otros referidos a la identidad profesional (0.72\%) y el prácticum (1.56\%). A su vez dentro del microtema de instrucción (segundo nivel) se hizo alusión a los aspectos de la planificación, desarrollo y evaluación de la sesión, siendo el desarrollo (47.7\%) el tema más frecuente (tercer nivel). El segundo macrotema: aprendizaje de los alumnos, representa un 25.09\% del total. Este macrotema se dividió en dos subtemas (segundo nivel): comportamiento de los alumnos en la sesión (21.95\%) y atención a la diversidad (3.13\%). Dentro del comportamiento de los alumnos en la sesión, el subtema atención de los alumnos (10.01\%) (tercer nivel) fue el más frecuente, Por último, los temas relacionados con contenidos académicos representan el $10.25 \%$ del total. Dentro de este tercer macrotema se hacía referencia al dominio del conocimiento (4.41\%) como un aspecto fundamental para impartir las sesiones, así como la importancia de desarrollar actividades (6.60\%).

Por último, en los resultados del análisis proposicional se observó que las tres condiciones generaron comentarios muy similares, obteniendo un 38\% en la reflexión individual y un 31\% en la reflexión entre compañeros y con el tutor. En el conocimiento narrativo, es decir, los recuerdos y las valoraciones, se obtuvo una mayor frecuencia (71\%) en las tres reflexiones. En cambio, se obtuvieron un menor número en las reglas y artefactos, conocimiento inferencial (24\%), y en el conocimiento explicativo (4\%), es decir, justificaciones prácticas y teóricas.

\subsection{Condiciones de reflexión}

Las pruebas no paramétricas de Chi-cuadrado permitieron comparar las tres condiciones para ver cuál de ellas permite la elaboración de conocimientos pedagógicos más sofisticados (conocimientos inferenciales frente a narrativos).

Comparación 1: Reflexión con compañero vs. Reflexión con tutor. La reflexión con compañero arrojó un balance de un $89.3 \%$ de conocimiento narrativo (75) y sólo un $10.7 \%$ de conocimiento inferencial (9). Por su parte la reflexión con tutor fue de un $71.8 \%$ (61) vs. 28.2\% (24) (ver figura 1).

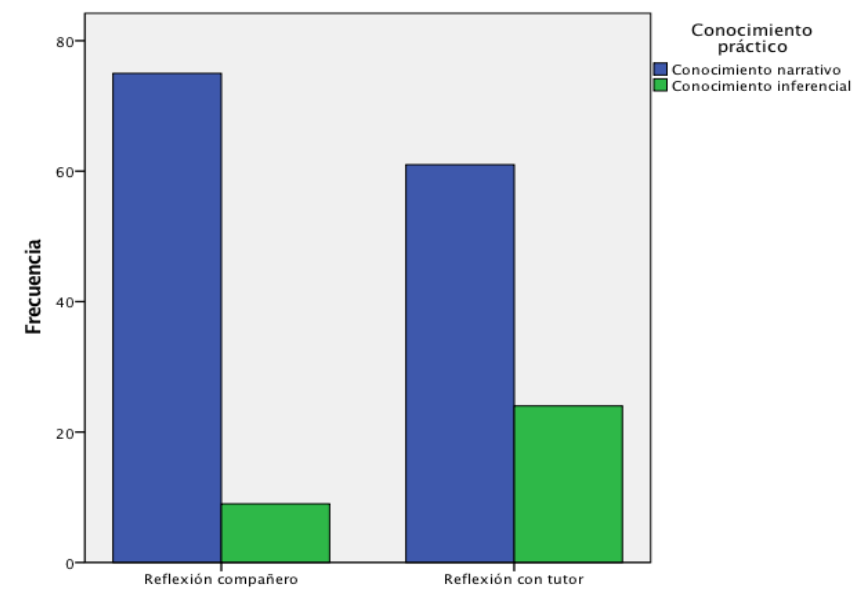

Figura 1. Distribución de conocimiento narrativo e inferencial en las condiciones de reflexión con compañero y reflexión con tutor 
Sin embargo, sí existen diferencias significativas entre la reflexión con compañero y la reflexión con tutor $x^{2}=8.254(p=0.004 ; g l=1)$ siendo esta última más beneficiosa a la hora de inferir reglas y artefactos de la práctica. V de Cramer de 0.421 , teniendo un efecto medio casi alto.

Comparación 2: Reflexión individual vs. Reflexión con tutor. Como se aprecia en la figura 2, un total de 67 unidades (71.3\%) de la reflexión individual destapó conocimiento narrativo mientras que sólo 27 (28.7\%) fue inferencial. Esta distribución no cambia con la ayuda del tutor, quien provoca que el profesor en formación redescriba su práctica a modo de recuerdos y valoraciones 61 (71.8\%) mientras que el conocimiento inferencial representa 24 (28.2\%).

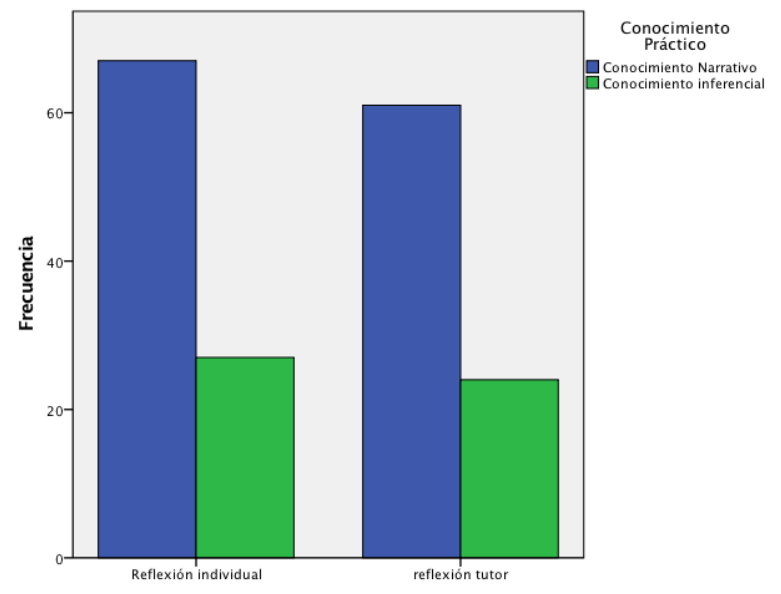

Figura 2. Distribución de conocimiento narrativo e inferencial en las condiciones de reflexión individual y con tutor

Se puede apreciar que no existen diferencias estadísticamente significativas $(p>0.05 ; g l=1)$ entre la reflexión individual y con tutor a la hora de generar conocimientos más sofisticados de la práctica. En este caso el valor de Chi cuadrado es $x^{2}=24.2(p=0.94)$. De modo más ajustado se puede ver cómo la $V$ de Cramer (Cohen, 1998) arroja un valor de 0.05, lo que indica un tamaño del efecto bajo.

Comparación 3: Reflexión individual vs. Reflexión con compañero. La reflexión individual arrojó un balance de un $71.3 \%$ de conocimiento narrativo (67 proposiciones) y sólo un $28.7 \%$ de conocimiento inferencial (27 proposiciones). Por su parte, la reflexión con compañero fue de un 89.3\% (75 proposiciones) frente a un $10.7 \%$ (9 proposiciones) (ver figura 3). Sí existen diferencias significativas entre la reflexión individual y con compañero, ya que $x^{2}=8.917, g l=1$ con $p=0.003$. La V de Cramer es de 0.224. 


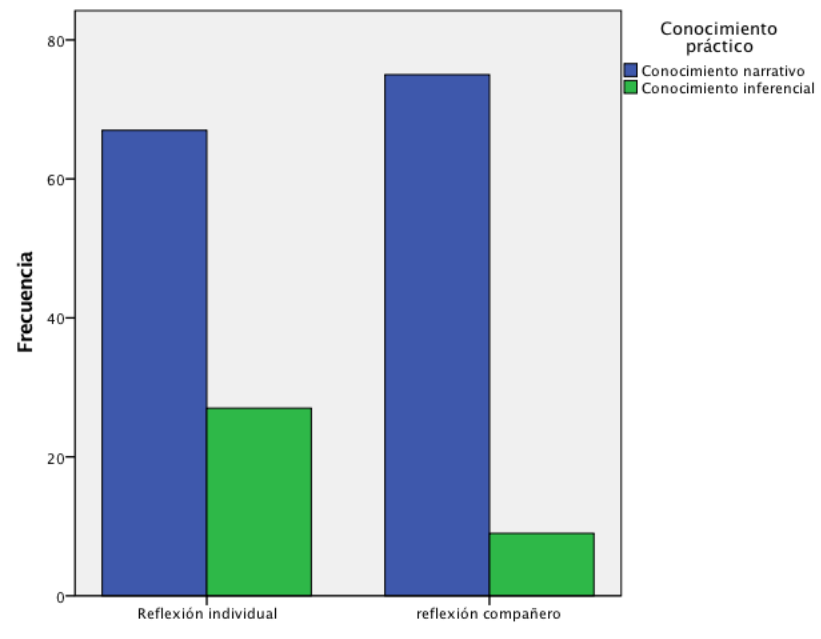

Figura 3. Distribución de conocimiento narrativo e inferencial en reflexión la individual y con compañero

Las comparaciones realizadas se realizaron con el conocimiento narrativo e inferencial, ya que no hay casos para comparar las justificaciones (teóricas y prácticas). Ninguna de las tres condiciones (individual, con compañero y con tutor) supera el $1 \%$, al ser más difícil encontrar razones para explicar las acciones realizadas durante la sesión de acuerdo a su propia experiencia (justificaciones prácticas) o teorías educativas (justificaciones teóricas).

\subsection{Implicaciones}

La investigación educativa deja cada vez más en claro que la formación de los docentes es un elemento clave para la mejora de la enseñanza, siendo uno de los aspectos más relevantes en la adquisición de conocimientos prácticos sobre las experiencias concretas (Cuevas, 2013). Lo que se pretende con este estudio es comprobar qué tipo de conocimiento pedagógico surge de la práctica docente mediante el uso de un análisis triple: análisis de incidentes críticos, análisis temático y análisis proposicional. Este análisis resulta beneficioso para valorar un posible camino en los procesos de formación del profesorado en la práctica, ya que la analiza en distintos niveles, de lo más general a lo más concreto.

El análisis de incidentes críticos proporciona información sobre los aspectos que los alumnos en prácticas consideraban más importantes, tanto los aspectos positivos como los negativos. Con este primer análisis los alumnos observaron lo que hacían bien y los aspectos que consideraban que debían mejorar, dónde habían encontrado dificultades, qué había sido lo más difícil, etc. Todos ellos forjan la base de la experiencia docente propia que permite mejorar acciones futuras.

El análisis temático nos proporciona una información más detallada del contenido de la sesión. El tema del que más se habla es el que se refiere al desarrollo de la sesión; sin embargo, el tema menos mencionado son las competencias básicas. Esto puede explicarse porque tienen más información sobre cómo desarrollar una sesión que sobre las competencias básicas, tema reciente por la modificación de la ley educativa.

Por último, el análisis proposicional ayuda a entender el tipo de conocimiento práctico que se revela en la acción. Observando los resultados se comprueba que los alumnos que reflexionaron con su tutor aprendieron un conocimiento más complejo y generalizable, es decir, reglas y artefactos para poder aplicar en sus clases, mientras que con las reflexiones individuales y con compañeros comprobaron qué habían hecho durante su sesión, es decir, valoraciones y recuerdos. Las pruebas de Chi cuadrado mostraron diferencias significativas entre ambas condiciones, con un efecto medio (casi alto) según la $V$ de Cramer. En cambio, no existen diferencias entre la reflexión individual y con tutor a la hora de generar conocimientos inferenciales, la $V$ de Cramer indica un efecto bajo. Si bien es verdad que en otras investigaciones el tipo de actividad y las conversaciones con el profesor-tutor representan importantes 
fuentes de información para que el profesor en formación ejecute con efectividad las tareas asociadas a la docencia y se haga cargo de su propio aprendizaje (Ugartetxea, 2002), Gairín, Feixas, Guillamón y Quinquer (2004) añaden que la tutoría a través del feedback sobre la práctica ayuda a los profesores en formación a aprender de sus errores, encontrar solución a los posibles problemas, alcanzar los objetivos, consolidar sus aprendizajes y aplicar principios abstractos a otros contextos.

Este estudio precisa que la información relevante que el tutor comparte con el profesor en formación sucede, por regla general, en un plano narrativo y no tanto en uno inferencial. Es decir, las conversaciones giran en torno a recordar y evaluar lo que se hizo en la práctica, no tanto en extraer principios y reglas de la misma. Tejada-Fernández, Carvalho-Dias y Ruiz-Bueno (2017) deja entrever esta misma idea cuando arguye que los procesos de mentoría que se llevan a cabo en los centros escolares suelen ser de intercambio de información y no de reflexión sobre la práctica. Esto induce a pensar que la promesa del prácticum debe favorecer el perfil de un profesional reflexivo que "...sea capaz de indagar en su práctica" (Tejada-Fernández et al., 2017, p. 109) no siempre se cumple en el sentido más profundo del término.

Los resultados de este estudio nos hacen entender la importancia que juega el tipo de reflexión a la hora de extraer conocimientos más sofisticados de la práctica. Una reflexión mediada (con compañero o tutor) permite extraer generalizaciones de la práctica con más facilidad, lo cual puede ayudar a identificar mejor los problemas de la práctica, mejorar su comprensión e intervenir sobre ello de forma más eficaz.

\section{Conclusiones}

La tutoría en educación se centra en la caracterización de las prácticas de enseñanza en relación con los ámbitos de competencia, las relaciones interpersonales y el desarrollo del conocimiento (Eraut, 2004).

Nuestra propuesta se basa en la divulgación de las ideas que han surgido en las conversaciones de tutoría y buscar si hay algún contenido que sea de apoyo para mejorar la práctica de los docentes en cuanto a las estrategias de enseñanza.

El uso de una guía para la reflexión ayudaría en el desarrollo de un conjunto común de criterios (directrices) para la supervisión práctica en los programas universitarios.

En conclusión, esta investigación postula que los estudios futuros deben tener en cuenta dos aspectos: el conocimiento de la práctica por medios científicos y el cambio de la práctica atendiendo a las necesidades de los maestros, por ejemplo, a través de la creación de instrumentos o herramientas comprensibles y transmisibles que puedan ser utilizadas por los profesionales en sus entornos escolares.

No obstante, es necesario señalar que la muestra de participantes fue baja como para poder hacer una generalización de los resultados a otras prácticas docentes, por lo que sería conveniente extender este análisis a otros programas de formación para comprobar la consistencia de los resultados y la usabilidad en otros escenarios educativos.

Existe, por otro lado, una limitación en el tiempo que transcurre entre la recogida de datos y el análisis, ya que la recogida de datos se hizo en dos momentos diferentes (2013 y 2014) y el análisis se elaboró en el 2015. No obstante, dentro de los objetivos no se ha contemplado valorar los posibles efectos de aprendizaje, sino describir los aprendizajes de los profesores en formación.

La tercera limitación a tener en cuenta es que sólo se realizó una grabación por sesión de clase, y cada alumno decidió en qué momento realizarla. Sería conveniente grabar una sesión al comienzo del prácticum, reflexionar sobre ella, y grabar otra sesión los últimos días para comprobar el avance de los conocimientos pedagógicos con el transcurso de la experiencia docente durante las prácticas.

La cuarta limitación evidencia que las comparaciones realizadas han sido sólo con el conocimiento narrativo e inferencial, ya que no hubo casos para comparar las justificaciones (teóricas y prácticas), pues ninguna de las tres condiciones supera el $1 \%$. 


\section{Agradecimiento}

Los datos presentados en esta investigación fueron extraídos y analizados al amparo del programa Lifelong Learning de la Unión Europea, número de referencia 526318-LLP-1-2012-1-EE-COMENIUS-CMP.

\section{Referencias}

Beltrán, J. (2003). Estrategias de aprendizaje. Revista de Educación, 332, 55-73.

Binda, N. U. y Balbastre-Benavent, F. (2013). Investigación cuantitativa e investigación cualitativa: buscando las ventajas de las diferentes metodologías de investigación. Revista de Ciencias Económicas, 31(2), 179-187. Recuperado de

https://revistas.ucr.ac.cr/index.php/economicas/article/view/12730

Carnot, M. J. y Stewart, D. (2006). Using concept maps in college level psychology and social work clases. En A. J. Cañas y J. D. Novak (Eds.), Proceedings of the second conference on concept mappings (pp. 292-295). San José, Costa Rica. Recuperado de http://cmc.ihmc.us/cmc2006Papers/cmc2006-p232.pdf

Cerezo, C. R., Ortega, F. Z. y Molina, F. Z. (2016). La autonomía y orientación en el Espacio Europeo de Educación Superior mediante el portafolio y la tutoría. Estudios sobre Educación, 19, 261-268. Recuperado de https://www.unav.edu/publicaciones/revistas/index.php/estudios-sobre-educacion/article/view/4622

Clandinin, D. J. (1985). Classroom practice: teacher images in action. Londres: Falmer.

Cohen, J. (1988). Statistical power analysis for the behavioral sciences (2a. ed.). Hillsdale, NJ: Lawrence Erlbaum Associates.

Cohen, L. y Manion, L. (1994). Research methods in education (4a. ed.) Londres: Routledge.

Cuevas de la Garza, M. S. (2013). La docencia universitaria a través del conocimiento profesional práctico: pistas para la formación. Sinéctica, 41, 2-18. Recuperado de https://sinectica.iteso.mx/index.php/SINECTICA/article/view/29

Dewey, J. (1933). How we think: a restament of the relation of refective thinking to the deductive process. Boston, MA: Heath and Company.

Elbaz, F. (1981). The teacher's "practical knowledge": report of a case study. Curriculum Inquiry, 11(1), 4371. doi: $\underline{10.1080 / 03626784.1981 .11075237}$

Elliott, J. (1993). El cambio educativo desde la investigación-acción. Madrid: Morata.

Eraut, F. (2004). Transfer of knowledge between education and workplace settings. En H. Rainbird, A. Fuller y A. Munro (Eds.), Workplace learning in context (pp. 201-221). Londres: Routledge.

Fernández, R., Gértrudix, F., De Cisneros, J., Rodríguez, J. y Rivas, B. (2015). La formación del profesorado en Tecnología Educativa: prácticas profesionales. Revista Latinoamericana de Tecnología Educativa, 14(1), 115-132. doi:10.17398/1695-288X.14.1.115

Gairín, J., Feixas, M., Guillamón, C. y Quinquer, D. (2004). La tutoría académica en el escenario europeo de la Educación Superior. Revista Interuniversitaria de Formación del Profesorado, 18(1), 61-7. Recuperado de https://www.aufop.com/aufop/revistas/arta/impresa/45/350 
Grau, S., Gómez, C. y Perandones, T. (2009). La formación del profesorado como factor decisivo de la excelencia educativa. Recuperado de http://rua.ua.es/dspace/bitstream/10045/13199/1/PROPUESTAS\%20CAP.\%201.pdf

Hatton, N. y Smith, D. (1995) Reflection in teacher education: towards definition and implementation. TeaChing and Teacher Education, 11(1), 33-49. doi:10.1016/0742-051X(94)00012-U

Husu, J., Toom, A. y Patrikainen, S. (2008). Guided reflection as a means to demonstrate and develop student teachers' reflective competencies. Reflective Practice: International and Multidisciplinary Perspectives, 9(1),37-51. doi:10.1080/14623940701816642

Johnson, B. (1995). Why conduct action research? Teaching and Change, 3(2), 90-104. ERIC: EJ520875

Kemmis, S. y McTaggart, R. (1988). Cómo planificar la investigación-acción. Barcelona: Laertes.

Kieras, D. E. y Bovair, S. (1984). The role of a mental model in learning to operate a device. Cognitive science, 8(3), 255-273. Recuperado de http://hdl.handle.net/2027.42/24767

Koballa, T. R. y Glynn, S. M. (2007). Attitudinal and motivational constructs. En S. K. Abell y N. G. Lederman (Eds.), Handbook of research on science education (pp. 75-102 Mahwah, NJ: Lawrence Erlbaum Associates.

Korthagen, F. (1999). Linking reflection and technical competence: the logbook as an instrument in teacher education. European Journal of Teacher Education, 22, 191-207. doi:10.1080/0261976899020191

Korthagen, F. y Lagerwerf, B. (2001). "Teachers' professional learning: how does it work?" En F. A. J. Korthagen, J. Kessels, B. Koster, B. Lagerwerf y T. Wubbels, Linking practice and theory: the pedagogy of realistic teacher education (pp. 175-206). Mahwah, NJ: Lawrence Erlbaum Associates.

Mena, J., García, M., Clarke, A. y Barkatsas, A. (2016). An analysis of three different approaches to student teacher mentoring and their impact on knowledge generation in practicum settings. European Journal of Teacher Education, 39(1), 53-76. doi:10.1080/02619768.2015.1011269

Mena, J., García, M., Gómez, R. y Ramírez, S. (2016). La importancia de las sesiones de tutoría en la elaboración del conocimiento profesional docente en el practicum: reflexiones sobre un modelo de análisis del discurso. En M. A. Flores, M. L. Carvalho y C. Silva (Eds.), Formação e aprendizagem profissional de professores: contextos e experiências [Formación y aprendizaje profesional de profesores: contextos y experiencias (pp. 77-107). Portugal: De Facto.

Mena, J., Henissen, P. y Loughran, J. (2017). Developing pre-service teachers' professional knowledge of teaChing: the influence of mentoring. TeaChing and Teacher Education, 66, 47-59.

doi:10.1016/i.tate.2017.03.024

Mena, J., Sánchez, E. y Tillema, H. (2008). Analysis of the in-service teachers' spontaneous reflections. What is said and what is done. Teachers and Teaching: Theory and Practice, 14(2), 95-114.

Mishra, P. y Koehler, M. J. (2006). Technological pedagogical content knowledge: a framework for teacher knowledge. Teachers College Record, 108(6), 1017-1054. Recuperado de

https://www.tcrecord.org/content.asp?contentid=12516

Orland-Barak, L. (2005). Portfolios as evidence of mentors' learning: what remains 'untold'. Educational Research, 47(1), 25-44. doi:10.1080/0013188042000337541

Raposo, M. y Zabalza, M. A. (2011). La formación práctica de estudiantes universitarios: repensando el practicum. Revista de Educación, 354, 17-20. Recuperado de http://www.revistaeducacion.educacion.es/re354/re354.pdf 
Rodríguez, J. (2001). Análisis de tablas de contingencias. Estadística Informática: casos y ejemplos con el sPSS. Recuperado de https://rua.ua.es/dspace/bitstream/10045/8139/1/CONTINGENCIA.pdf

Schön, D. A. (1983). The reflective practitioner. Nueva York: Basic Books.

Serrat, P. M. y Badia, M. C. (2015). Profesorado novel y feedback del tutor: un estudio de casos. Profesorado: Revista de Currículum y Formación del Profesorado, 19(1), 405-421. Recuperado de https://recyt.fecyt.es/index.php/profesorado/article/view/41045

Shulman, L. (2002). Forgive and remember. The challenges and opportunities of learning from experience. LaunChing the next generation of new teachers Symposium Proceedings. Santa Cruz, CA: New Teacher Centre at the University of California.

Strauss, A. y Corbin, J. (1994). Grounded theory methodology. En N. K. Denzin y Y. S. Lincoln (Eds.), Handbook of qualitative research (pp. 273-285). Thousand Oaks, CA.

Tejada-Fernández, J., Carvalho-Dias, M. L. y Ruiz-Bueno, C. (2017). El prácticum en la formación de maestros: percepciones de los protagonistas. Magis. Revista Internacional de Investigación en Educación, 9(19), 91-114. doi:10.11144/Javeriana.m9-19.pfmp

Tierney, R. J. y Mosenthal, J. H. (1983). Cohesion and textual coherence. Research in the Teaching of English, 17, 215-229.

Toom, A. (2006). Tacit pedagogical knowing. At the core of teacher's professionality. University of Helsinki. Recuperado de http://ethesis.helsinki.fi/julkaisut/kay/sovel/vk/toom/tacitped.pdf

Toom, A. (2012). Considering the artistry and epistemology of tacit knowledge and knowing. Educational Theory, 62, 621-640. doi:10.1111/edth.12001

Ugartetxea, J. (2002). La metacognición, el desarrollo de la autoeficacia y la motivación escolar. Revista de Psicodidáctica, 13, 49-74. Recuperado de https://www.ehu.eus/ojs/index.php/psicodidactica/article/view/135

Zeichner, K. (2007). Accumulating knowledge across self-studies in teacher education. Journal of Teacher Education, 58(1), 36-46. doi:10.1177/0022487106296219 


\section{Anexo}

Tabla I. Análisis temático (efectuado)

\begin{tabular}{|c|c|c|c|c|}
\hline $\begin{array}{l}\text { Positivo/ } \\
\text { negativo }\end{array}$ & Theme & Unidad de pensamiento & $\begin{array}{c}\text { Incidente } \\
\text { Crítico }\end{array}$ & $\begin{array}{c}\text { Tipo de } \\
\text { conocimiento práctico }\end{array}$ \\
\hline Negativo & TPC & $\begin{array}{l}\text { E: La última servía para evaluar, entonces por } \\
\text { eso decidí seguir, y como ellos estaban ahí } \\
\text { trabajando en sus murales, no sé qué, pues } \\
\text { nos les iba a cortar. }\end{array}$ & 41.1 & Justificación práctica \\
\hline Positivo & TPC & $\begin{array}{l}\text { T: me ha parecido bien algunas modificaciones } \\
\text { que has hecho, por ejemplo de la presentación } \\
\text { porque se podía alargar demasiado }\end{array}$ & 41.1 & Valoración positiva \\
\hline
\end{tabular}

Contexto: Una profesora en formación reflexiona con su tutora sobre la sesión de Geometría. TPC: Relación didáctica (entre el profesor y el aprendizaje del estudiante).

Incidente Crítico: X.1. (positivo); X.2. (negativo)

Tabla II. Análisis proposicional (extracto)

\begin{tabular}{|c|c|c|c|c|c|c|}
\hline \multirow[t]{2}{*}{ Transcripción } & \multirow[t]{2}{*}{ Episodios } & \multirow[t]{2}{*}{ Ciclos } & \multirow[t]{2}{*}{ Turnos } & \multirow[t]{2}{*}{ Proposiciones } & \multicolumn{2}{|c|}{$\begin{array}{c}\text { Conocimiento } \\
\text { Práctico }\end{array}$} \\
\hline & & & & & Tipo & SA/ST \\
\hline $\begin{array}{l}\text { MAESTRA: Yo, lo mismo que te } \\
\text { digo la mayoría de las veces, que } \\
\text { muy bien trabajado, que bien } \\
\text { buscada la información, que bien } \\
\text { buscado el material, que bien } \\
\text { motivado, o sea que me gustó } \\
\text { mucho. También hay que decir } \\
\text { que la clase se ha portado un } \\
\text { poquito mejor de lo normal... } \\
\text { JONATHAN: Sí, un poquito. Yo } \\
\text { creo que un poquito bastante, le } \\
\text { imponía ahí... }\end{array}$ & & & 2 & $\begin{array}{l}\text { P.1. Muy bien trabajado } \\
\text { P.2. Bien buscada la } \\
\text { información } \\
\text { P.3. [Bien buscado] el material } \\
\text { P.4. [Bien] motivado } \\
\text { P.5. Me gustó mucho } \\
\text { P.6. La clase se ha portado un } \\
\text { poquito mejor de lo normal } \\
\text { P.7. \{Se han portado\} un } \\
\text { poquito bastante }\{\text { mejor\} }\end{array}$ & $\begin{array}{l}\text { AP+ } \\
\text { AP+ } \\
\text { AP+ } \\
\text { AP+ } \\
\text { AP+ } \\
\text { AP+ } \\
\text { AP+ }\end{array}$ & $\begin{array}{l}\text { SA } \\
\text { SA } \\
\text { SA } \\
\text { SA } \\
\text { SA } \\
\text { SA } \\
\text { ST }\end{array}$ \\
\hline
\end{tabular}

Contexto: Un profesor en formación reflexiona con su tutora de prácticas sobre la sesión que ha realizado. Episodios: EV=Evaluación; AP+=Valoraciones positivas; SA/ST= Maestra/Profesor en prácticas 